\title{
El consumo alimentario: abordaje teórico-crítico en la interfase economía, salud y ambiente
}

\author{
Food Consumption: Critical Theoretical Approach in the \\ Economy, Health, and Environment Interface
}

O consumo alimentício: abordagem teórica e crítica na área de interação entre economia, saúde e ambiente

\author{
Mónica Izurieta Guevara \\ Universidad Andina Simón Bolívar, Sede Ecuador. Quito, Ecuador \\ monica.izurieta@uasb.edu.ec \\ https://orcid.org/0000-0002-9890-677X
}

DOI: https://doi.org/10.32719/25506641.2022.11.8

Recibido: 15 de enero de 2021 - Revisado: 6 de abril de 2021

Aceptado: 19 de mayo de 2021 - Publicado: 1 de enero de 2022

Artículo de investigación

Licencia Creative Commons

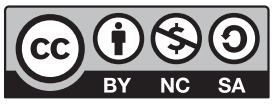




\section{Resumen}

El objetivo de esta investigación es aportar a la discusión del consumo de alimentos a través de un abordaje novedoso en la interfase transdisciplinaria de la economía, salud y ambiente. Para ello, el consumo de alimentos es explicado, desde un enfoque teórico, en el marco interpretativo de la determinación social de la salud. Así, desde la dinámica actual del régimen alimentario corporativo, se identifican procesos críticos del consumo de alimentos relacionados con la mercantilización y nocividad de las dietas, la ocupación del espacio para su distribución, la publicidad alimentaria y el lobby corporativo frente al Estado. Se explican las relaciones de estos procesos con la salud y enfermedad de los cuerpos y las expresiones en el ambiente. Con ello, se logra una mirada integral del consumo de alimentos que supera a los postulados de la soberanía del consumidor y al enfoque reduccionista de las ciencias de nutrición y dietética; ambos campos hasta hoy limitados a una abstracción del hecho alimentario en un plano individual.

Palabras clave: consumo, alimentación, sustentabilidad, responsabilidad social empresarial, salud.

JEL: Q01 Desarrollo sostenible.

\section{Abstract}

The purpose of this research is to contribute to the discussion of food consumption using an innovative approach in the transdisciplinary interface of the economy, health, and the environment. To this end, food consumption is explained from a theoretical perspective, in the interpretive framework of the social determination of health. Thus, based on the current dynamics of the corporate food regime, we have identified critical food consumption processes related to the commercialization and harmfulness of diets, the occupation of space for distribution, food advertising, and corporate lobbying towards the state. The relationships of these processes with the health and illness of the bodies and the expressions in the environment are hereby explained. This provides a comprehensive view of food consumption that surpasses the claims of consumer sovereignty and the reductionist approach of nutrition and dietetic sciences; both fields limited until this date to an abstraction of the reality of food at an individual level.

Keywords: consumption, food, sustainability, corporate social responsibility, health.

JEL: Q01 Sustainable development.

\section{Resumo}

O objetivo desta pesquisa é o de aportar à discussão do consumo de alimentos através de uma abordagem original na área de interação transdisciplinar entre economia, saúde e ambiente. Para tal, o consumo de alimentos é explicado, com um enfoque teórico, no marco interpretativo da determinação social da saúde. Deste modo, desde a dinâmica atual do regime alimentar corporativo identificam-se processos críticos no consumo de alimentos relacionados com a mercantilização e nocividade das dietas, a ocupação do 
espaço para sua distribuição, a publicidade alimentar e o lobby corporativo frente ao Estado. Explicam-se as relações destes processos com a saúde e a doença dos corpos e suas expressões no ambiente. Assim sendo, obtém-se uma visão integral do consumo de alimentos que supera os postulados da soberania do consumidor e o enfoque reducionista das ciências da nutrição e da dietética, ambos campos, até hoje limitados a uma abstração do fato alimentar no plano individual.

Palavras-chave: consumo, alimentação, sustentabilidade, responsabilidade social empresarial, saúde.

JEL: Q01 Desenvolvimento sustentável.

\section{Introducción}

E

n este ensayo se interpreta el consumo de alimentos en la interfase economía, ambiente y salud utilizando como recurso el modelo de la determinación social de la salud (Breilh 2014; 2020) y a partir de una mirada crítica al régimen alimentario corporativo (McMichael 2009), actual forma del sistema alimentario en la modernidad capitalista.

Frente a la actual crisis civilizatoria y ecológica, de la cual la producción global y local de alimentos hace parte, la transdisciplinariedad y el pensamiento crítico resultan útiles para superar la fragmentación de disciplinas que se relacionan con el hecho alimentario y proponer programas, proyectos y políticas integradoras que aborden a la realidad integrando las partes en un todo, evitando perderse en un bosque de intervenciones desconectadas y ajenas a las dinámicas materiales y subjetivas propias de los territorios. Uno de los debates actuales sobre alimentación y salud es la ineficacia de la política pública al no lograr solucionar la escalada de la silenciosa epidemia de sobrepeso y obesidad y tampoco terminar con la desnutrición infantil. Al tiempo de esta discusión, se ha colocado sobre la mesa de análisis la necesidad de discutir y actuar frente a la necesidad de dietas sustentables en el afán de que los sistemas alimentarios no perjudiquen los ecosistemas. Los enfoques teóricos clásicos de la economía (postulados de la soberanía del consumidor) y las ciencias de nutrición y dietética podrían estar limitados para responder a estos desafíos por su abordaje reduccionista y fragmentado que desboca en política pública que se discute solo a un nivel individual de la realidad en la alimentación.

En este sentido, se propone una nueva aproximación al consumo de alimentos a través de un análisis teórico-crítico que da cuenta de las relaciones, jerarquías y dimensiones conectadas entre la economía, salud y ambiente, desde un lente crítico y una postura transdisciplinaria. Se procura responder a la pregunta: ¿cómo interpretar 
el consumo alimentario desde un enfoque crítico transdisciplinario en los campos de la economía, ambiente y salud?

En este ensayo, se utiliza como método una revisión de literatura relevante, principalmente teórica, que sustenta la articulación de disciplinas alrededor del objeto de estudio, los argumentos teóricos se expresan en algunas evidencias centradas en América Latina. El ensayo, respondiendo a su carácter académico, está estructurado en tres partes: esta introducción, el desarrollo argumentativo y la conclusión.

En primera instancia, se presenta al objeto de estudio: el consumo alimentario en el contexto de la modernidad capitalista. Posteriormente, se expone la necesidad de alternativas transdisciplinarias para transiciones sustentables y saludables en el campo de la producción y consumo de alimentos. Se explica la relación entre economía y empresa con los procesos ambientales y los de salud y enfermedad a través del movimiento de subsunción de lo biológico en lo social, que tiene como base los argumentos de Jaime Breilh (1994; 2020) y Nancy Krieger (2015) en su desarrollo de la epidemiología crítica. Con ello, se propone la discusión de cuatro procesos críticos del consumo de alimentos desde una jerarquía general de la realidad alimentaria y su articulación con las jerarquías particular e individual. Con esta aproximación, se procura superar la acostumbrada discusión que se reduce solamente al plano de ingesta de alimentos y enfermedades. Hacia el final de esta interpretación, se explica el metabolismo de estos procesos alimentarios integrados a una realidad de consumo que es socialmente determinada y posee un carácter metabólico con la naturaleza.

Para concluir, se realiza una abstracción de los principales elementos del análisis crítico propuesta para el objeto de estudio, el consumo de alimentos, y se recogen las ventajas de esta interpretación frente a los abordajes clásicos de la economía y la nutrición.

\section{El consumo alimentario: abordaje teórico-crítico en la interfase economía, salud y ambiente}

\section{La interpretación del consumo de alimentos como desafío}

Como correlato de la actual crisis civilizatoria y ecológica se encuentran la producción y consumo de alimentos por su marcada incidencia en la salud y el ambiente. Por un lado, la producción de alimentos a gran escala provoca contaminación de suelos, agua y deforestación de grandes extensiones de bosques; mientras tanto, el consumo de cierto tipo de alimentos y bebidas ocasiona epidemias aún incontrola- 
das como el sobrepeso y la obesidad. Paradójicamente, la situación de desnutrición y hambre en varios rincones del planeta no se ha solucionado, aun tras décadas del discurso y práctica dominante de la productividad agrícola (revolución verde).

El abordaje del consumo alimentario se ha desarrollado principalmente desde el enfoque de la soberanía de consumidor de la economía clásica y desde las ciencias de la nutrición y dietética de forma fragmentada. Ambos campos adolecen de una debilidad trascendental para la interpretación del consumo de alimentos: la abstracción hacia un plano netamente individual de la realidad. Entonces, desde el pensamiento económico convencional, se asume a un consumidor racional, autónomo en cuanto a sus preferencias y elecciones, quien satisface sus necesidades en un mercado supuestamente en supuesto autocontrol. Desde el campo de la nutrición, las dietas son asunto del paciente o de su familia y lo que importa es dividir platillos en quantums adecuados de ingesta calórica.

Las limitaciones de estos abordajes clásicos del consumo alimentario repercuten en algunos traspiés que imposibilitan cambios y soluciones a la crisis de los sistemas alimentarios. Se exponen algunos:

- Los procesos de consumo se asumen desvinculados de una jerarquía más amplia de la realidad en la que se vislumbran las condiciones del modelo económico y civilizatorio imperante.

- Se minimiza la importancia del análisis histórico como insumo para comprender la conformación del proceso alimentario en una sociedad.

- Se ignoran las relaciones de poder que están inmersas en la configuración de mercados de alimentos y las condiciones de inequidad hacia productores locales que juegan en el mercado con estructuras organizacionales de pequeñas y medianas empresas o con ferias tradicionales.

- Se pierde de vista las características propias de grupos sociales diferenciados que se encuentran inmersos y responden frente a la complejidad de un proceso económico civilizatorio específico; la cultura y gastronomía de sus territorios; los desafíos actuales de la maternidad y paternidad precarizada por el trabajo informal y el trabajo doméstico; los procesos migratorios campo ciudad así como país a país; la gestión y acceso al tiempo libre y alimentación en la vida cotidiana; la capacidad de generar procesos alimentarios saludables desde la asociatividad y el cooperativismo.

- El abordaje de metabolismo sociedad y naturaleza queda suspendido por la noción de que lo ecológico en la alimentación es asunto de otros campos y el canon instrumentalista de la economía clásica.

En suma, el consumo de alimentos puede y debe asumirse como un objeto de estudio complejo, multidimensional y de múltiples relaciones entre campos disci- 
plinarios, relaciones que permitirían explicar los procesos de consumo alimentario en su integralidad económica, espacial, social, ecológica, biológica y metabólica. El de la gestión no está desligado, pues es elemento central del diseño y ejecución de acciones que permitirían una regeneración de condiciones de equidad en la producción, circulación y consumo de alimentos, así como una política pública eficaz a los desafíos de salud y ambiente. Argumentos que rayan en el mismo sentido, aunque con diferentes tintes, críticos o no, pueden encontrarse en la edición de 2018 de la FAO, UNICEF y del Programa Mundial de Alimentos: The Sate of Food Security and Nutrition in the Wolrd y en los informes de la comisión de The Lancet: Global Syndemic de 2019 (Swinburn et al. 2019).

\section{La necesidad de alternativas transdisciplinarias}

Frente a la crisis de la modernidad capitalista, el empeño de la autora - y seguramente el de muchos otros, mujeres y hombres - es comprender realidades, participar y gestionar acciones hacia el cambio. Los axiomas de la teoría clásica de la economía no responden a este desafío. El afán de crecimiento económico sin límites, como postulado clave del pensamiento económico ortodoxo, ha sido por demás refutado frente a la crisis social, ambiental, sanitaria y alimentaria a la que nos enfrentamos en casi todos los rincones del planeta. Pueden consultarse las exposiciones desarrolladas por decenas de autores desde las nuevas escuelas de economía crítica y economía ecológica quienes sustentan la antinomia que representa el crecimiento económico frente a la sobrevivencia en nuestro planeta. Citaré uno de ellos por su fortaleza teórica y empírica en la demostración de las "ilusiones y realidades del crecimiento" como él mismo anota. Thomas Piketty $(2013,643)$ en su obra El Capital en el siglo XXI, en un trabajo que le tomó a él y a su equipo investigador quince años, recoge datos de países de distintos continentes y construye principalmente la evolución de la producción, el ingreso y riqueza mundial a partir de la revolución industrial. Concluye:

La lección general de mi investigación es que la evolución dinámica de una economía de mercado y de propiedad privada que es abandonada a sí misma contiene en su seno fuerzas de convergencia importantes, relacionadas sobre todo con la difusión del conocimiento y de calificaciones, pero también poderosas fuerzas de divergencia, potencialmente amenazadoras para nuestras sociedades democráticas y para los valores de justicia social en que están basadas [...] La principal fuerza desestabilizadora se vincula con el hecho de que la tasa de rendimiento privado del capital $r$ puede ser significativa y duraderamente más alta que la tasa de crecimiento del ingreso y la producción $g$. [...] el pasado devora al porvenir. (Énfasis añadido) 
Es necesario comprender que la vigencia del pensamiento económico convencional es una barrera para acciones integradoras y eficaces. En el campo de la alimentación, tampoco hay excepciones, puesto que se sigue interpretando a la realidad alimentaria desde un reducido plano individual y, lo que es peor, desvinculado de la determinación económica, ecológica, espacial y cultural que da forma a diferentes patrones de consumo alimentario y que responde, además, a un devenir histórico.

Revertir la tendencia de la educación superior, que nos forma como especialistas de un campo y profanos de otros, es tarea necesaria para las transformaciones integrales que requiere la supervivencia de la humanidad. Es además una respuesta ética-científica para las próximas generaciones con quienes estamos en deuda. En este sentido, el economista José Manuel Naredo $(2013,133)$ aporta:

Estas disciplinas ya no pueden seguir siendo mundos separados, que razonen con lógicas supuestamente autónomas sobre el poder y el dinero, sino que han de conectarse abrazando enfoques transdisciplinarios y multidimensionales. $\mathrm{Y}$ en tal caso, al trascender los reduccionismos propios del dinero y el poder, la toma de decisiones políticas o económicas de cierta entidad ya no podría cerrarse con razonamientos técnico-científicos en el seno de las propias disciplinas.

Los postulados económicos clásicos generan opacidades al momento de objetivar la realidad, aun peor, facilitan a los profesionales un ethos que valora solamente lo pragmático e instrumental en el campo de la economía y la empresa. En este caminar entre lo caduco y lo vitalmente necesario, en la transición hacia la construcción de tiempos y espacios de vida, se coloca un elemento clave: la transdisciplinariedad para las ciencias económicas y administrativas. Es necesario promover una conciencia potenciada por la plenitud de conceptos y percepciones de la realidad compleja que pueda armonizar las partes con el todo. Aproximarnos a las arbóreas posibilidades de un paradigma crítico de la economía a través de su encuentro con los campos de la salud y el ambiente, permitiría el logro de transiciones eficaces.

La definición de transdisciplinariedad de Miguel Martínez $(2007,16)$ resulta interesante: "conocimiento superior emergente, fruto de un movimiento dialéctico de retro y pro alimentación del pensamiento, que nos permite cruzar los linderos de diferentes áreas del conocimiento disciplinar y crear imágenes de la realidad más completas, más integradas y, por consiguiente, también más verdaderas".

Mirar las partes en función del todo, poder iluminar las relaciones con conciencia integradora, asumir la complejidad en la interpretación de la realidad, es un camino eficaz a la hora de gestar política económica, organizaciones, innovaciones, proyectos y programas. Para las transformaciones civilizatorias necesarias, el cami- 
no transdisciplinario en la economía y la gestión ha de superar la visión instrumentalista atosigada por el pensamiento económico clásico.

\section{La interfase en economía, ambiente y salud}

Necesitamos enrumbar nuestra genialidad hacia una economía que se enfoque en lo vital para evitar una muerte a gotas del género humano y de la naturaleza de la que somos indivisibles (Izurieta 2020). Allí donde brota la vida, en la naturaleza, la salud y los cuidados, hay que trabajar mancomunadamente desde la ciencia y la praxis.

Lo transdisciplinario en la economía, ambiente y salud colectiva encuentra un correlato en la construcción de la epidemiología crítica latinoamericana, específicamente en el modelo de la determinación social de la salud de Jaime Breilh (2014; 2020). El siguiente análisis se enfoca en la articulación del campo económico con el de ambiente y salud de acuerdo con la mencionada propuesta.

Se propone comprender a la salud como una categoría polisémica y multidimensional. Desde esta noción ontológica y, con base en una lógica dialéctica, se identifican procesos saludables o protectores de la vida y procesos destructores o nocivos. Estos dos procesos contrarios se desarrollan en tres dimensiones de la realidad: general (sociedad), particular (grupos) e individual (fenotipo y genotipo y psiquis de los cuerpos). En segundo lugar, se entiende a la salud como una construcción subjetiva imbricada en los modos de vida propios de grupos sociales diferenciados por su clase social, género, etnia. Estos sujetos sociales perciben y responden estratégicamente y con resiliencia subjetiva a la determinación y reproducción económica y social de sus sociedades.

El carácter nocivo de la forma económica actual, según Breilh, reproduce una forma malsana de civilización y modos de vida que incide en los procesos de salud y enfermedad. Este es el encuentro del campo de la economía y la salud. Esta forma civilizatoria, determinada por la lógica de concentración de capitales, tiene un carácter de metabolismo sociedad y naturaleza que le corresponde. Aquí el encuentro de la economía, salud y ambiente. A continuación, en la figura 1, interpretada por la autora con base en el modelo mencionado, tiene como intención repensar el enfoque en el que tradicionalmente miramos a la economía, esa forma lineal de extracción, producción y consumo que opaca nuestro entendimiento de la realidad en el campo de la economía y empresa. 
Figura 1

\section{Articulación de procesos económicos, ambientales y de salud}

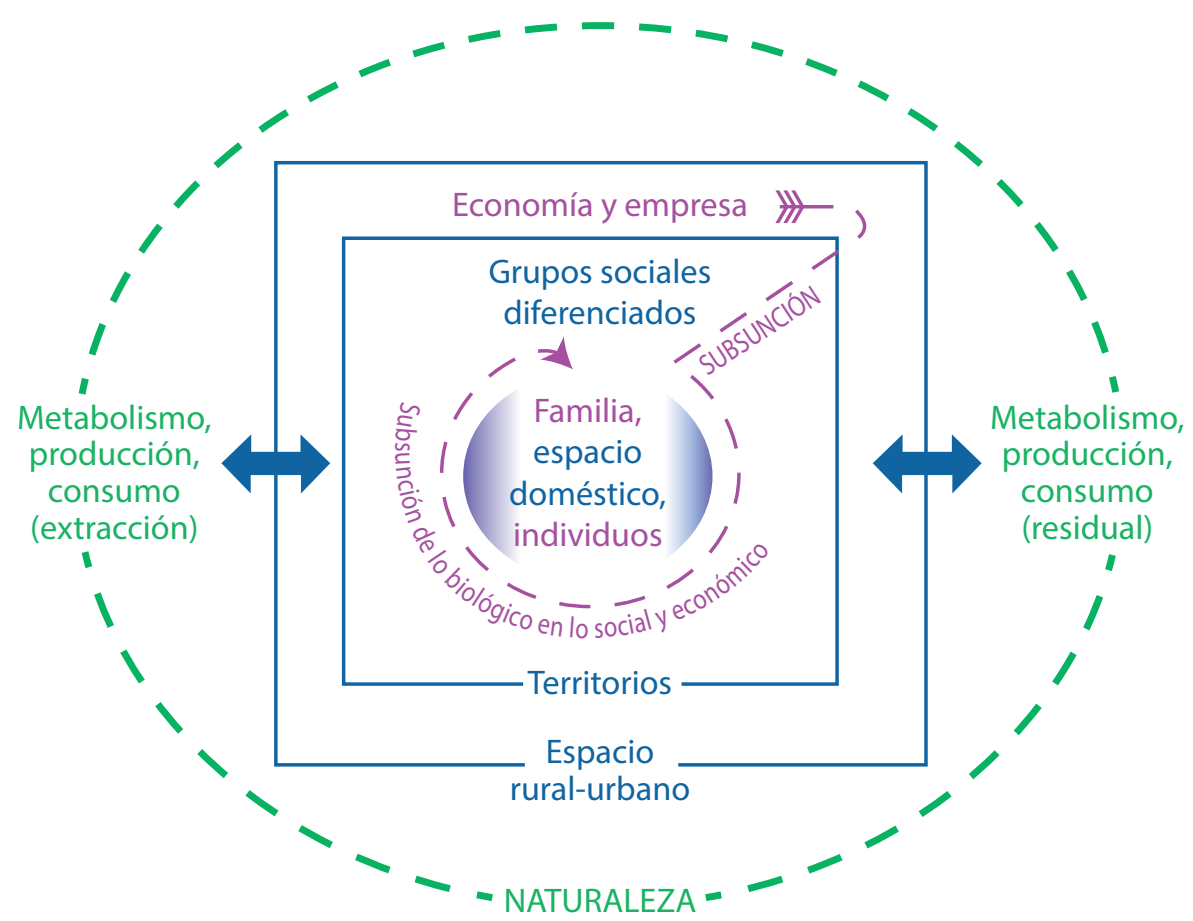

Elaboración propia.

Al siglo XXI, varios estudiosos de la economía crítica, desde el lente de pensamiento latinoamericano, han renovado la categoría subsunción (representada en línea entrecortada azul de la figura) que proviene de la palabra aufhebung, del alemán, que significa "arrastrar arriba lo que está debajo" y poner "dentro lo que estaba fuera" (Dussel 2014, 73-74) y fue, originalmente, utilizada por Karl Marx en su obra El Capital (1975) para expresar la subordinación formal y real en el trabajo. Esta categoría, actualizada a la dimensión de consumo, ha sido retomada por Andrea Santos (2018) quien se refiere al movimiento de subsunción como la adecuación de la estructura material de la producción y el consumo a la lógica de valorización del capital. Por otro lado, Álvaro García (2009) señala que las fuerzas subjetivas e inte- 
lectivas del ser humano también se incorporan al beneficio del capital. El mexicano Jorge Veraza (2018) asume la subsunción real de consumo como la subordinación de fuerzas productivas procreativas a favor de la acumulación de capitales y en detrimento de la naturaleza.

En el campo de la salud, este movimiento de subsunción coloca lo biológico intrincado en la jerarquía más amplia de la forma económica-social. Para entender este movimiento de subsunción de lo biológico en lo social (también representado en la figura 1), es necesario retomar los aportes de Nancy Kreiger (2005) quien estableció el concepto de embodiment para entender cómo los seres humanos incorporamos, biológicamente, lo material, económico y social del mundo en que vivimos. Breilh (1994, 80; énfasis añadido) también señaló que: "no se puede entender la relación social-biológico si no se asume la subsunción de lo biológico en lo social, y no se puede comprender dicha incorporación si no se interpretan los hitos del devenir histórico de cada sociedad y las transformaciones de los territorios".

Así pues, el movimiento que deviene de la forma económica predominante tiene dos momentos: una subsunción de las dimensiones de producción y consumo desde la jerarquía general, y una subsunción de lo biológico en lo social que se concreta en el nivel individual y que expresa los procesos de enfermedad, fenotipos, genotipos y psiquis de los seres humanos: los embodiments. De acuerdo con las formas predominantes de producción y consumo de cada sociedad $-\mathrm{y}$ esto se relacionada directamente con economía y empresa-, existen expresiones en la naturaleza que reflejan nuestro intercambio orgánico con ella.

\section{El consumo de alimentos en la interfase economía, salud y ambiente: procesos críticos}

De acuerdo con Andrea Santos $(2014,11)$ "la modificación en un patrón de consumo alimentario supone cambios en los hábitos, costumbres, necesidades y referencias de los individuos, así como de modificaciones en la estructura productiva, comercial y de generación y distribución de la riqueza". En contraposición con la teoría de soberanía del consumidor de la microeconomía ortodoxa, se plantea que estos cambios no se derivan únicamente de la demanda o las preferencias alimenticias de consumidores aparentemente soberanos. Se debe entender que, desde una jerarquía más amplia, la configuración de la oferta, existen procesos de condicionamiento en la producción y consumo de alimentos que mercantilizan las dietas y las adecuan a la lógica de valorización del capital corporativo, en el movimiento llamado como subsunción real de consumo, lo cual, repercute en el dominio del espacio para la circulación de los ali- 
mentos. Se sabe que el acaparamiento de los mercados globales sucede desde apenas 20 corporaciones a nivel mundial (Ottero, Pechlaner y Gürcan 2015).

Entender el devenir de los objetos de estudio desde una perspectiva histórica es importante puesto que se pueden esgrimir las causas de las transformaciones. En el caso del consumo alimentario, esta perspectiva histórica permite entender por qué las dietas se han transformado en nocivas para seres humanos y ambiente. Por ello, $\mathrm{y}$ aunque no es objetivo de este ensayo, se debe mencionar brevemente que los patrones de consumo alimentario de finales de siglo XX e inicios del XXI se configuran en el denominado Régimen alimentario corporativo, ${ }^{1}$ denominado así por Philip McMichael (2009) quien señala que la estructura del régimen actual de alimentación está sustentada en una estrategia corporativa global basada en una nueva división internacional del trabajo agrícola al tiempo que una tensión central que yace entre la globalización de la agroindustria y los movimientos de productores y consumidores que claman por una soberanía alimentaria. Para Gerardo Otero (2013) existe, además, una caracterización específica al este tercer régimen: la injerencia corporativa alimentaria en los gobiernos, el incremento de oligopolios de agroempresas multinacionales y el desarrollo de la biotecnología.

En la figura 2 se interpreta el consumo de alimentos desde tres jerarquías: general, particular e individual, situadas en el momento histórico del régimen alimentario corporativo.

\section{La jerarquía general del consumo alimentario: \\ procesos de determinación}

En este nivel, operaría la forma económica dominante del régimen alimentario corporativo en cuanto génesis de los procesos críticos que mercantilizan la dieta y la adecuan a la acumulación privada del capital. A partir de 1980, esta lógica de mercantilización de las dietas adquiere además una nocividad diferenciada de períodos anteriores que obedece a una acelerada conformación de oligopolios de empresas productoras y comercializadoras de los alimentos y bebidas procesadas. Al respecto, pueden citarse autores latinoamericanos como Gerardo Otero (2015) y su análisis de la dieta neoliberal, así como el análisis teórico y empírico sobre modificaciones de los patrones de consumo alimentario en México, de Andrea Santos (2014).

1. Le anteceden el régimen liberal colonialista marcado aproximadamente entre 1870 y 1930 , el régimen de posguerra en los años 1950 a 1970 y el neoliberal hasta 1980, desde la actualidad, de acuerdo con la perspectiva histórica de los regímenes alimentarios de Harriet Friedman y Philip McMichael. 
Figura 2

\section{Interpretación de los patrones de consumo en la interfase economía, salud y ambiente}

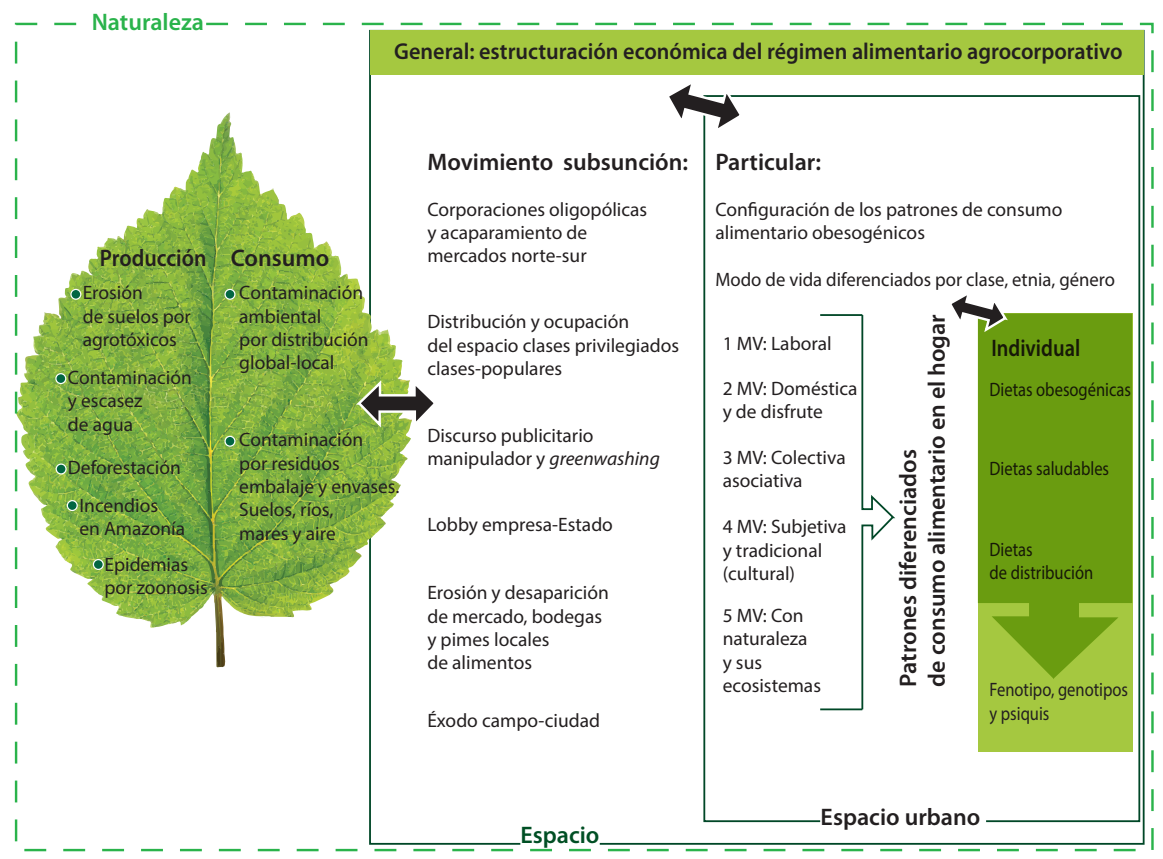

Elaboración propia.

Conforme con las dinámicas actuales de la producción y consumo de alimentos en la modernidad capitalista, y de acuerdo con la integración de campos disciplinarios que asume la determinación social de la salud (Breilh 2020), la autora de este ensayo propone que en el nivel general se analicen los siguientes procesos críticos: a) concentración oligopólica en la producción y distribución de alimentos y acaparamiento de mercados en detrimento de pymes, negocios familiares, mercados y ferias tradicionales; b) ocupación del espacio público y privado para la distribución de dietas mercantilizadas y nocivas; c) publicidad alimentaria y manipulación en la percepción de los alimentos y bebidas; y d) injerencia de corporaciones en política pública.

Estos procesos tendrían la capacidad de incidir en la configuración de los mercados alimentarios con características más o menos homogéneas: crecimiento de 
la circulación de alimentos y bebidas procesadas, proliferación de autoservicios en varios formatos y con horarios extendidos, desaparición paulatina de los mercados y bodegas tradicionales de pequeñas familias (Santos 2014; Hollenstein 2019).

En un espacio alimentario, como el de un barrio latinoamericano en plena transición urbana, los mercados alimentarios proliferan en medio de una multitud de contradicciones: las fuerzas de lo tradicional y lo moderno, lo familiar y corporativo, lo público y privado; fuerzas que se encuentran y definen la oferta de alimentos a los hogares. Las formas de alimentación de estos hogares también suceden en medio de contradicciones entre lo protector y destructor; subsunción estructural, autonomía familiar y asociativa; transiciones demográficas propias del siglo XXI; precarización del trabajo y de la maternidad; resiliencia migratoria campo-ciudad o entre país propio-país ajeno.

Los alimentos y bebidas correspondientes a esta forma de consumo, propias del régimen alimentario corporativo, son nocivos en cuanto a su contenido material por el nivel de artificialización. Para contrarrestar este carácter de nocividad de las dietas, el proceso de la publicidad alimentaria la subvierte a través de imágenes y discursos manipuladores que puede explicarse como una forma de producción semiótica (Echeverría 2009). La estrategia de manipulación discursiva e icónica de la publicidad de alimentos y bebidas procesadas parte justamente del nivel general hacia la configuración de representaciones del alimento en consumidores.

Paralelamente, el lobby corporativo hacia los gobiernos de turno y ministerios de economía, agricultura y salud acompañan a los otros procesos mencionados. Es una estrategia propia del régimen alimentario corporativo el influenciar en el Estado a favor de sus empresas productoras y distribuidoras de alimentos, táctica ampliamente documentada por científicos y activistas como Marion Nestle (2012), en su obra Food Politics; Gerardo Ottero (2013; 2015), en sus desarrollos del Régimen alimentario neoliberal y la dieta neoliberal; Michael Simons (2006), en sus publicaciones referidas a corporaciones alimentarias y su intervención en gobiernos, academia y programas sociales.

Todos estos procesos conformarían un movimiento de subordinación (subsunción) estructural — desde el nivel general — según la propuesta de epidemiología crítica y el modelo de la determinación social de la salud (Breilh 2014; 2020). Este movimiento encuentra su contrario: el de autonomía, constituido por la agroecología, movimientos y cooperativas de consumo consciente que parten de estrategias asociativas y organizaciones barriales urbanas y rurales, en un nivel particular del modelo. 
La jerarquía particular del consumo alimentario: modos de vida y configuración de patrones de consumo

Sidney Mintz argumenta que las estrategias de poder son estructurales y reproducen las condiciones circunstanciales o los términos en los cuales la gente obtiene sus alimentos y cambian sus hábitos alimentarios, lo cual puede configurarse en un nivel particular del modelo de la determinación social de la salud (Mintz citado en Santos 2014, párr. 3). Se debe tener en cuenta que ese movimiento de subsunción del consumo alimentario al capital agroalimentario corporativo, que sucede de lo general a lo particular, no es igual para todos los sujetos consumidores pues los expone y vulnera a unos más que otros; por lo tanto, los procesos de salud y enfermedad dependerán de la caracterización del modo de vivir del grupo al que pertenece el sujeto consumidor y las relaciones de poder que atraviesan su clase, etnia y género.

La categoría modos de vida (Breilh 2003) —señalado como "MV" en la figura 2- está conformada por cinco dimensiones: trabajo, consumo, relaciones colectivas y asociativas, subjetividad e identidad y relacionamiento con la naturaleza. Estas dimensiones representan eslabones para la configuración de las dietas en los hogares, las cuales suceden entre los procesos de determinación versus protectores autónomos (participación en grupos de consumo, agricultura urbana, dietas sanas y sustentables) y conforman patrones de consumo alimentario diferenciados para cada grupo social analizado. Se invita a pensar en que existe un modo distinto de alimentarse para cada clase social y que también influyen en la dieta de los grupos la etnia y gastronomía de los territorios. Por otro lado, pertenecer a una asociación o cooperativa de consumo responsable de alimentos puede modificar la dieta familiar hacia patrones más saludables de consumo; ser conscientes del alimento como don de la naturaleza, conocer y practicar agricultura urbana podría mejorar la calidad de la dieta. Entonces, la determinación social y económica del consumo alimentario atraviesa nuestros modos de vida — nivel particular-y, por lo tanto, emergen diferencias en los procesos de salud y enfermedad.

La jerarquía individual del consumo alimentario: los embodiments

En esta jerarquía se concreta la subsunción de lo biológico en lo social. Regresando a Krieger (2011), se argumenta que el proceso de embodiment implica una consideración que va más allá del fenotipo y genotipo y es superior a una vaga definición del ambiente en interacción con los genes de una población. Krieger (2011, 22) señala: "vivimos encarnándonos". Esta encarnación es más que el resultado de las condiciones sociales incorporadas bajo la piel, es un proceso más interactivo y 
recíproco de materia corporal y psique combinadas, individual y colectivamente en el mundo biofísico. Los tipos diferenciados de embodiment de las dietas son determinados desde la forma económica dominante y la estructura del régimen alimentario específico que atraviesan los modos de vida de grupos adscritos a clases sociales y etnias diversas.

El caso de México es ilustrador: desde la ejecución del Tratado de Libre Comercio América del Norte (TLCAN), el país inició un proceso de sustitución de su dieta tradicional. Así, para 2009 se importaba el $95 \%$ de la soya, $80 \%$ del arroz, $56 \%$ del trigo, $33 \%$ del maíz y el $40 \%$ de la carne. También se convirtió en el primer importador de leche en polvo. En suma, para 2018 se importaba el $46 \%$ de los alimentos (Blanco-García y Florence 2016). El síntoma más perceptible de estos cambios estructurantes en el sistema alimentario mexicano fue el abandono de la dieta centrada en el maíz y frijoles, sustituida por bebidas azucaradas y trigo, alimentos procesados con alto contenido de carbohidratos (Santos 2014).

Actualmente, México ocupa el segundo lugar a nivel mundial en la prevalencia de obesidad en adultos con un $32,4 \%$, muy cerca de Estados Unidos $(38,2 \%)$. La proyección para 2030 es que el $39 \%$ de la población mexicana será obesa (García y Ledezma 2018). De 1980 a 2000, se identificó en este país un incremento del 47\% en la mortalidad por diabetes mellitus tipo 2, pasando de ser la novena causa de mortalidad en 1980 a la tercera en 1997, y segunda causa de mortalidad a nivel nacional en 2010. Esos son los fenotipos y genotipos originados por un cambio de dieta en el contexto del TLCAN (Santos 2014).

Ecuador es un país de reflexiones interesantes. La Encuesta Nacional de Salud y Nutrición (2012) demostró que la anemia y el retraso de crecimiento en menores de 5 años continuaban con prevalencias de 26 y $25 \%$ respectivamente. A la vez, el sobrepeso y obesidad alcanzaron un $8,6 \%$ en este grupo de edad (valor duplicado con relación al último estudio de 1986). En los escolares de 5 a 11 años se registró una prevalencia alarmante del $30 \%$ de sobrepeso y obesidad y en los adultos de 20 a 60 años este indicador alcanzó el $63 \%$ (Freire et al. 2014).

Se mencionó que los embodiments de la dieta pueden variar por clase social y etnias. Por ejemplo, en Estados Unidos los índices de sobrepeso y obesidad en adultos son más altos en aquellos Estados de mayor inequidad y vulnerabilidad de clases populares (TaeEung 2018). Los niños mexicanos y afroamericanos en Estados Unidos muestran una mayor prevalencia de obesidad comparada con niños no hispanos y blancos, la relación es del doble en esta prevalencia (Ogden et al. 2012). En este país, la prevalencia aumenta en niños de edad escolar de población latina (26\%) y afrodescendientes $(23 \%)$ mientras que la prevalencia de obesidad más baja se encuentra en niños no hispanos cuyos padres terminaron el colegio (TaeEung 2018). 
La explicación de estos fenómenos no se deriva de un determinismo biológico, tampoco de libres elecciones de consumo. Existiría una determinación económica, social y espacial para enfermar.

\section{Metabolismo de régimen agroalimentario corporativo y naturaleza}

Desde que tenemos contacto con la tierra para nuestra sobrevivencia hemos concretado un intercambio orgánico con la naturaleza. Alfred Schmidt (1977), en su obra El concepto de naturaleza en Marx, afirma que la producción es siempre social y consiste en una apropiación de la naturaleza dentro y a través de una forma social, que el trabajo concreto presupone un sustrato natural y las relaciones sociales están mediadas por naturaleza y viceversa. Se trata de un intercambio que sucede principalmente en el momento de producción de la economía. En los sistemas alimentarios este intercambio de seres humanos y naturaleza deviene nocivo en cuanto la producción se torna extractivista e intensiva, problema evidente a escala global. La configuración de patrones alimentarios en el siglo XXI se diferencia de cambios en anteriores regímenes por su correlato de destrucción ambiental que parece fuera del control de la gestión empresarial y pública (Santos 2014).

A breves rasgos, se podría anotar la confluencia de los siguientes procesos críticos en el metabolismo de régimen alimentario corporativo y naturaleza: monocultivos y uso intensivo de pesticidas que erosionan los suelos, motivan la deforestación de bosques y manglares; el agua contaminada o envasada y mercantilizada a gran escala saca fuentes naturales; la crianza industrial de animales para consumo de carne avícola, porcina y vacuna requiere del uso intensivo de antibióticos y hormonas y se proveen de extensos monocultivos de maíz y soja transgénicos para alimentación de animales. Estas son expresiones que resultan de una forma concreta de economía, sus dinámicas de producción y consumo y sus sistemas alimentarios.

Alrededor del mundo son cada vez más las personas en riesgo por la ingesta de metales en agua y alimentos, las emisiones y desechos industriales, la concentración de uso de fertilizantes, la acumulación de estos en las algas de los mares que posteriormente son ingeridas por los peces que servirán de comida para los seres humanos. Los metales pesados se acumulan en tejidos y órganos de los animales pasando después a la producción de carne, leche o huevos (Reyes et al. 2016).

Por otro lado, los desafíos frente a la problemática del agua y los sistemas alimentarios no son un asunto menor. El consumo de agua a nivel mundial se ha sextuplicado desde 1940; si la tendencia de consumo y contaminación continúa, para 
2025 más de la mitad de la humanidad sufrirá escases de agua (Sacher 2006). La contaminación del agua se refiere a formas de producir alimentos: nitratos producto de la fertilización excesiva para el cultivo de suelos, la exposición a aguas residuales, tanto industriales como domésticas; contaminación por plaguicidas; escorrentía procedente de las zonas de pastoreo y corrales de engorde de animales, son algunas de las prácticas que afectan el estado y disposición de agua (FAO 1997).

El uso de agrotóxicos es una sombra gigantesca que vulnera a productores y consumidores en los sistemas alimentarios. Los estudios en Brasil muestran una importante evidencia en cuanto a los riesgos de su uso en los cultivos de alimentos. La tercera parte de los alimentos consumidos en la cotidianidad brasileña está contaminada con agrotóxicos, de estos, un $30 \%$ con elementos no autorizados para su cultivo y/o que rebasan los límites máximos de residuos. En la agricultura, agrotóxicos y fertilizantes químicos producen intoxicación en los trabajadores, neoplasias y malformaciones al tiempo que la vida de los suelos se pierde y se pierden las especies que en este habitan (Carneiro 2015).

En Ecuador se puede citar la situación del banano. La producción de banano y plátano se intensifica con la entrada de la revolución verde. Las plagas que padecía el banano por causa del sistema de producción (monocultivo) llevó a incrementar una gran cantidad de agrotóxicos en el campo: "entre 1972 y el 2002, el Ecuador incrementó 47 veces la compra de plaguicidas, y los gastos de importación de estos insumos pasaron de 45 a 116 millones de dólares" (Galarza Suárez 2019, 351). Respondiendo en parte a las condiciones climáticas del trópico, los productores ecuatorianos aplicaban en promedio entre 14 y 16 ciclos de fumigación al año mientras que la dosis recomendada para el uso de los fungicidas sistémicos aplicados era de máximo seis ciclos, señala Galarza Suárez. Estas prácticas indiscriminadas han afectado la biodiversidad marina perjudicando directamente a las personas que viven en las costas, donde su mayor fuente de proteína son los peces y los frutos del mar extraídos de forma artesanal.

Algo parecido sucede en Esmeraldas, provincia que concentra la mayor extensión de cultivo de palma africana — $66 \%$ del total de la superficie - y una tasa de crecimiento que refleja un incremento del 300\% solo en la década 2000-2010 (Paredes 2013). Solo por este cultivo "se estima que por cada tonelada de aceites se generan 2,5 toneladas de residuos contaminantes" (Movimiento Mundial por los Bosques Tropicales citado en Paredes 2013, 107-108), lo que provoca una disminución del número de especies de fauna y flora y ha perjudicado la salud de la población con manifestaciones como erupciones cutáneas y trastornos estomacales. Además, está la concentración de grasas y aceites en ríos y esteros del área circundante a las comu- 
nidades de La Chiquita y Guadalito que superan en un 200\% los límites permitidos (Paredes 2013).

\section{Conclusiones}

Para transitar a transformaciones sustentables y saludables es necesario un enfoque transdisciplinario y asumir a la realidad en su total complejidad, de tal manera que se tiendan puentes hacia economías de lo vital. En este ensayo se ha discutido el abordaje del consumo de alimentos que supera la abstracción que procuran los postulados clásicos de soberanía del consumidor, las nociones de la motivación y comportamiento del consumo y la ilusoria representación de los mercados de competencia perfecta con capacidad de autorregulación. Las ciencias de la nutrición tampoco resuelven este enfoque fragmentado, por considerar el hecho alimentario, principalmente, asunto de los individuos y sus familias y de su ingesta alimenticia.

Este ensayo, desde el enfoque transdisciplinario y crítico, que asume a la complejidad como reto de integración del todo con las partes y las partes con el todo, ha pretendido interpretar el consumo de alimentos como una realidad integrada a jerarquías generales, particulares e individuales que interaccionan con base en los movimientos de subsunción real de consumo y subsunción de lo biológico en lo social y, solo a partir de ello, comprender las expresiones de enfermedad en cuerpos $\mathrm{y}$ en el ambiente. Se asume al consumo alimentario como determinado por procesos económicos, espaciales, sociales y semióticos que configuran patrones de consumo que, a su vez, y de forma dialéctica entre jerarquías, expresan enfermedad o salud, dependiendo de la autonomía y procesos asociativos protectores frente a procesos destructores más generales. Se invita al lector a mirar esta propuesta como una forma, tal vez aún incompleta, pero absolutamente ética en el afán de contribuir a las ciencias económicas y administrativas como puentes para transitar hacia la defensa de la vida en el planeta, hacia formas económicas justas que procuren la dignidad de seres humanos, de los animales y la permanencia de los ecosistemas.

\section{Referencias}

Blanco-García, Ilian, y Theodore Florence. 2016. "Prácticas alimentarias desde una perspectiva sistémica completa”. Razón y Palabra 20 (3): 134-147. https://bit.ly/2WOe86L. 
Breilh, Jaime. 1994. "Las ciencias de la salud pública en la construcción de una prevención profunda: determinantes y proyecciones". En Las ciencias de la salud pública en la construcción de una prevención profunda: determinantes y proyecciones, editado por María Isabel Rodríguez, 63-100. Washington D. C.: Organización Panamericana de la Salud.

---. 2003. Epidemiología crítica: ciencia emancipadora e interculturalidad. Buenos Aires: Universidad Nacional de Lanus.

---. 2014. "Epidemiología crítica latinoamericana: raíces, desarrollos recientes y ruptura metodológica. La determinación social de la salud como herramienta de ruptura hacia la nueva salud pública-salud colectiva". En Tras las huellas de la determinación, editado por Carolina Morales y Juan Carlos Eslava, 17-67. Bogotá: Universidad Nacional de Colombia.

---. 2020. Critical Epidemiology and the People's Health. Nueva York: Oxford University Press.

Carneiro, Fernando. Dossiê ABRASCO: um alerta sobre os impactos dos agrotóxicos na saúde. São Paulo: EPSJV/Expressão Popular.

Dussel, Enrique. 2014. 16 tesis de economía política. Ciudad de México: Siglo XXI.

Echeverría, Bolívar. 2009. La definición de la cultura. Ciudad de México: Itaca.

Food Agriculture Organization of United Nations (FAO). 1997. Lucha contra la contaminación agrícola de los recursos hídricos. Roma: FAO. http://bit.ly/3b13VXR.

FAO, UNICEF, WFP and WHO. 2018. The Sate of Food Security and Nutrition in the World. Roma: FAO.

Freire, Wilma, María José Ramírez, Philippe Belmont, María José Mendieta, Katherine Silva, Natalia Romero, Klever Sáenz, Pamela Piñeiros, Luis Fernando Gómez y Rafael Monge. 2014. Tomo I: Encuesta Nacional de Salud y Nutrición de la población ecuatoriana de cero a 59 años. ENSANUT-ECU 2012. Quito: Ministerio de Salud Pública / INEC.

Galarza Suárez, Lucía. 2019. “Tierra, trabajo y tóxicos: sobre la producción de un territorio bananero en la costa sur del Ecuador”. Estudios Atacameños 34: 341-364. https://doi. org/10.22199/issn.0718-1043-2019-0034.

García, Álvaro. 2009. "El núcleo fundante del desarrollo capitalista: subsunción formal y subsunción real de las fuerzas productivas objetivas, asociativas, subjetivas e intelectivas del ser humano bajo el capital”. En Forma valor y forma comunidad: aproximación teórico-abstracta a los fundamentos civilizatorios que preceden al Ayllu Universal, editado por Álvaro Linera, 141-201. La Paz: Traficantes de Sueños. https://bit.ly/378t3ed.

García, Martha, y Guillermo Bermúdez. 2016. "La neocolonización del paladar en las décadas recientes”. Razón y Palabra 20 (3): 106-18.

García, Lorena, y Jesús Ledezma. 2018. "La obesidad, un verdadero problema de salud pública persistente en México”. Journal of Negative and No Positive Results 8 (3): 643-654. https://bit.ly/3bt4KK9.

Grosse, Robert, Carlos Santos, Javier Taks y Stefan Thimmel. 2006. Las canillas abiertas de América Latina II. La lucha contra la privatización del agua y los desafios de una gestión participativa y sustentable de los recursos hídricos. Montevideo: Casa Bertolt Brecht. 
Hollestein, Patric. 2019. ¿Están en riesgo los mercados y ferias municipales? Quito: Universidad Central del Ecuador.

Izurieta, Mónica. 2020. "La oportunidad para repensar una economía de lo vital”. El Telégrafo. 13 de junio. https://bit.ly/3d7tN7e.

Krieger, Nancy. 2005. "Embodiment: A conceptual Glossary for Epidemiology". Journal of Epidemiology \& Community Health 59 (5): 350-355. https://bit.ly/3b4EGUw.

---. 2011. Epidemiology and the People's Health. Theory and Context. Nueva York: Oxford University Press.

Martínez, Miguel. 2007. “Conceptualización de la transdisciplinariedad”. Polis 16 (1): 1-20. http://bit.ly/3rHUV0u.

Marx, Karl. 1975. El capital: crítica a la economía política. (Libro 1: el proceso de producción del capital). Ciudad de México: Siglo XXI.

McMichael, Philip. 2009. “A Food Regimen Genealogy”. Journal of Peasant Studies 36 (1): 139-169.

Mintz, Sydney. 1995. "Food and its Relation to Concepts of Power". En Food and Agrarian Orders in the World-economy, editado por Philip McMichael, 3-14. Londres: Green Wood Press.

Naredo, José. 2013. "Ideología político-económica dominante y claves para un nuevo paradigma”. Economía Crítica 16 (2):108-143. https://bit.ly/3adw3ru.

Nestle, Marion. 2012. How the Food Industry Influences Food Nutrition and Health. Los Angeles: University of California Press.

Ogden, Cynthia, Margaret Carroll, Brian Kit y Katherine Flegal. 2012. "Prevalence of Obesity and Trends in Body Mass Index among US Children and Adolescents, 1999 2010". Jama 307 (5): 483-490. http://bit.ly/3u1Gdnh.

Otero, Gerardo. 2013. "El régimen alimentario neoliberal y su crisis: Estado, agroempresas multinacionales y biotecnología”. Antípoda 17: 49-78. https://doi.org/10.7440/antipoda17.2013.04.

Otero, Gerardo, Gabriela Pechlaner y Efe Can Gürcan. 2015. "The Neoliberal Diet: Fattening Profits and People". En The Routledge Handbook of Poverty in the United States, editado por Stephen Nathan Haymes, María Vidal de Haymes y Reuben Jonathan Millen, 472-479. Nueva York: Routledge.

Paredes, Martha. 2013. "Palma africana en el norte de Esmeraldas. Un caso de (in)justicia ambiental e insustentabilidad”. En Ecología política del extractivismo en América Latina, editado por Gian Carlo Delgado Ramos, 68-97. Buenos Aires: CLACSO.

Piketty, Thomas. 2014. El capital en el siglo XXI. Bogotá: Fondo de Cultura Económica.

Reyes, Yulieth, Inés Vergara, Omar Torres, Mercedes Díaz y Edgar González. 2016. “Contaminación por metales pesados: implicaciones en salud, ambiente y seguridad alimentaria". Revista Ingeniería, Investigación y Desarrollo 16 (2): 66-77.

Santos, Andrea. 2014. El patrón alimentario del libre comercio. Ciudad de México: UNAM / CEPAL. 
El consumo alimentario: abordaje teórico-crítico en la interfase economía, salud y ambiente

---. 2018. “Acumulación en el sector agroalimentario: más allá de la acumulación por desposesión. Contribuciones a partir del sometimiento real del maíz al capital”. Ideas y Debates 48 (1): 127-131. https://bit.ly/38C01T9.

Scmidt, Alfred. 1977. El concepto de naturaleza en Marx. Madrid: Siglo XXI.

Simon, Michel. 2006. Apetite for Profit. Nueva York: Nation Books.

Swinburn, Boyd, Vivica Kraak, Steven Allender, Vincent Atkins, Philip Baker y Jessica Bogard. 2019. "The Global Syndemic of Obesity, Undernutrition, and Climate Change: The Lancet Commission Report". The Lancet 393 (10173): 791-846. https://bit.ly/3765baT.

TaeEung, Kim. 2018. "Longitudinal Analyses of Socioecological Obesogenic Factors in a Large Sample of US Children". Tesis doctoral, Universidad de Indiana, Bloomington. http://bit.ly/3afgAXU.

Veraza, Jorge. 2008. Subsunción real del consumo al capital. Ciudad de México: Ítaca.

Wallace, Robert. 2016. Big Farms Make Big Flue. Dispatches on Infectious Disease Agribusiness. Nueva York: NYC Press. 